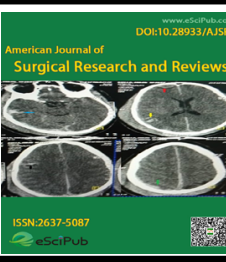

American Journal of Surgical Research and Reviews

(ISSN:2637-5087)

\title{
New Trends In Treacher Collins Syndrome: Bony Reconstruction And Regenerative Therapy
}

\author{
Luigi Clauser ${ }^{1}$, Chiara Gardin2, Letizia Ferroni ${ }^{2}$, Antonio Lucchi ${ }^{1}$, Carolina Sannino ${ }^{1}$, Maria \\ Elena de Notariis ${ }^{1}$ and Barbara Zavan ${ }^{2}$ \\ ${ }^{1}$ Unit of Maxillo-Facial Surgery, Istituto Stomatologico Italiano, Via Pace, 21, 20122 Milano, Italy; \\ ${ }^{2}$ Department of Biomedical Sciences, University of Padova, 35100 Padova, Italy
}

\section{ABSTRACT}

Aim:Treacher Collins syndrome is a rare congenital disorder of craniofacial development with a highly variable pheonotype. This syndrome occurs with an incidence of $1: 50,000$, and more than $60 \%$ of the cases have no previous family history and arise as the result of de novo mutations. The disorder displays an intricate underlying dysmorphology. Affected patients may suffer life-threatening airway complications and functional difficulties involving sight, hearing, speech, and feeding. Deformation of facial structures produces a characteristic appearance that includes malar-zygomatic hypoplasia, periorbital soft tissue anomalies, maxillomandibular hypoplasia, and ear anomalies. Management requires a specialized craniofacial team, as comprehensive care starts at birth and may require life-long follow-up. Standard craniofacial procedures for bony and soft tissue reconstruction are used. This article outlines current treatment strategies and future concepts for surgical and regenerative management. Methods:The new field of regenerative medicine and therapy offers the promise to improve some of these treatments. In particular, Structural Fat Grafting (lipostructure) seems to be a good strategy to restore the normal volume and contour of the face, and to provide a source of adipose-derived stem cells (ADSCs) with a multilineage differentiation potential. In this work, we present the case of a young girl with Treacher Collins Syndrome who underwent serial sessions of fat grafting in addition to other surgical bony reconstructive techniques. ADSCs have been isolated from the patient's lipoaspirate, and compared for their stemness properties with those of a healthy subject. Conclusion:Screening of the genome of the Treacher Collins patient using array-Comparative Genomic Hybridization (array-CGH) allowed us to identify some chromosomal imbalances that are probably associated with the syndrome. Correction of these imbalances and asymmetries by modulating ADSCs could be an innovative approach to improve and stabilize the results of the surgical treatment of Treacher Collin Syndrome.

Keywords:

Craniofacial surgery, Treacher Collins syndrome, regenerative medicine, structural fat grafting, adipose-derived stem cells, gene expression; array-CGH,tissue engineering

\section{*Correspondence to Author:}

Prof. Luigi Clauser Unit of MaxilIo-Facial Surgery, Istituto Stomatologico Italiano, Via Pace, 2120122 Milano, Italy, $\mathrm{Ph}+3902541761$

\section{How to cite this article:}

Luigi Clauser, Chiara Gardin, Letizia Ferroni, Antonio Lucchi, Carolina Sannino, Maria Elena de Notariis and Barbara Zavan. New Trends In Treacher Collins Syndrome: Bony Reconstruction And Regenerative Therapy. .American Journal of Surgical Research and Reviews, 2021, 4:22.

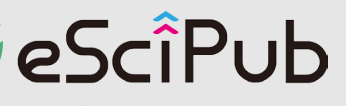
eSciPub LLC, Houston, TX USA. Website: https://escipub.com/ 


\section{INTRODUCTION}

Treacher Collins Syndrome (TCS; OMIM \#154500) ${ }^{[1]}$ is a rare autosomal dominant craniofacial malformation affecting 1:50,000 newborns ${ }^{[2]}$. This syndrome occurs during the fifth to eighth week of fetal development, and affects the proper formation of the first and second pharyngeal arches, leading to profound facial dysmorphism ${ }^{[3]}$. These pharyngeal arches are composed of mesenchymal cells derived from mesodermal and cranial neural crest cells, which give rise to a wide variety of facial structures including skeletal, muscular, and neural elements. It is thought that most craniofacial disorders, such as those associated with TCS, arise due to defects in the formation, proliferation, migration, and/or differentiation of neural crest cells ${ }^{[4]}$. The resulting phenotype of TCS, which is often bilateral and relatively symmetric, includes hypoplasia of the facial bones, particularly the zygomatic complex and mandible, cleft palate, and middle and external ear defects that result in conductive deafness ${ }^{[5]}$. Considerable phenotypic variability exists among patients, with the most severe cases leading to perinatal death due to respiratory distress, and mild cases that escape clinical diagnosis. Despite this large spectrum of variability, penetrance is thought to be nearly complete [6].

The gene responsible for TCS has been mapped to chromosome 5q32-q33.1 and named Treacher Collins-Franceschetti syndrome 1 gene (TCOF1; OMIM *606847) [7]. More than 130 different mutations have been identified spanning all 26 exons of the gene and these are responsible for over $90 \%$ of TCS cases ${ }^{[2]}$. The majority of mutations in TCOF1 are predicted to result in truncated treacle proteins. Treacle is a nucleolar phosphoprotein implicated in ribosomal DNA gene transcription and preribosomal RNA processing [8]. Mice haploinsufficient for Tcof1 exhibit diminished production of the mature $28 \mathrm{~S}$ subunit in neuroepithelial cells and neural crest cells. This results in nucleolar stress activation of p53 ${ }^{[9]}$.
Increased p53 levels lead to $\mathrm{G} 1$ cell cycle arrest and specific apoptosis of neuroepithelial cells, which results in hypoplastic neural crest-derived structures of the craniofacial skeleton [2].

Molecular analysis of the TCOF1 gene determined that $40 \%$ of newborns with TCS have inherited one mutated copy of TCOF1, whereas $60 \%$ of cases arise as the result of de novo mutations ${ }^{[10]}$. The extreme variability in the degree to which individuals can be affected, and the high rate of de novo mutations, makes the provision of genetic counseling extremely complicated. Furthermore, genetic screening for TCOF1 mutations during early gestation appears to be economically unviable except in families with a known history of TCS. Consequently, the majority of individuals with craniofacial abnormalities are detected during mid-to-late gestation through ultrasound screening [11]. Nevertheless, phenotypic diagnosis at this stage is extremely difficult, even with the most sophisticated ultrasonography available today. Therefore, at present, multiple reconstructive surgeries remain the only available treatment option for TCS management. Because of the complex interactions of the numerous and simultaneously affected tissues, care of individuals with TCS usually requires a multidisciplinary team approach. This approach may involve intervention from a number of healthcare professionals both pre and postoperatively, including craniofacial surgeons, orthodontists, ophthalmologists, otolaryngologists, and speech pathologists ${ }^{[12]}$. However, despite the multiple rounds of surgery that a TCS patient typically endures, they are rarely fully corrective in the long-term.

Regenerative medicine holds promise for new strategies to improve the treatment of this disorder. In this context, use of structural fat grafting (SFG) has increased in popularity in the last 20 years. First introduced as a method to improve facial aesthetics, SFG has become an effective and reproducible technique to treat some craniofacial malformations [13]. Several 
advantages are rapidly making SFG the preferred approach for facial augmentation.It is autologous and completely biocompatible, with a minimal risk of infection, and grafts are available in sufficient quantities in most patients, are naturally integrated into the host tissue, and have a natural appearance and feel that is distinctly better than that of implants and most fillers. In addition, long-term studies showed that these grafts can last for decades or longer [14]. Apart from these characteristics, SFG represents a delivery system of adipose-derived stem cells (ADSCs), which exhibit a multilineage differentiation potential and secrete angiogenic and antiapoptotic factors [15].

A case of a 6 year-old young patient with TCS treated over many years with different surgical reconstructive procedures is reported. SFG following Coleman's procedure ${ }^{[16]}$ was the first and the last surgical treatment. Gene expression analyses with real-time PCR were performed to compare the stemness properties of ADSCs isolated from the patient's lipoaspirate and those of a healthy subject. Possible chromosomal imbalances related to the disorder were investigated by array-Comparative Genomic Hybridization (array-CGH) analysis of genomic DNA of the TCS patient. Deep comprehension of ADSCs characteristics and their correlation with the genotype of the TCS patient could represent an innovative tool to improve the surgical treatment of cranio-maxillofacial deformities.
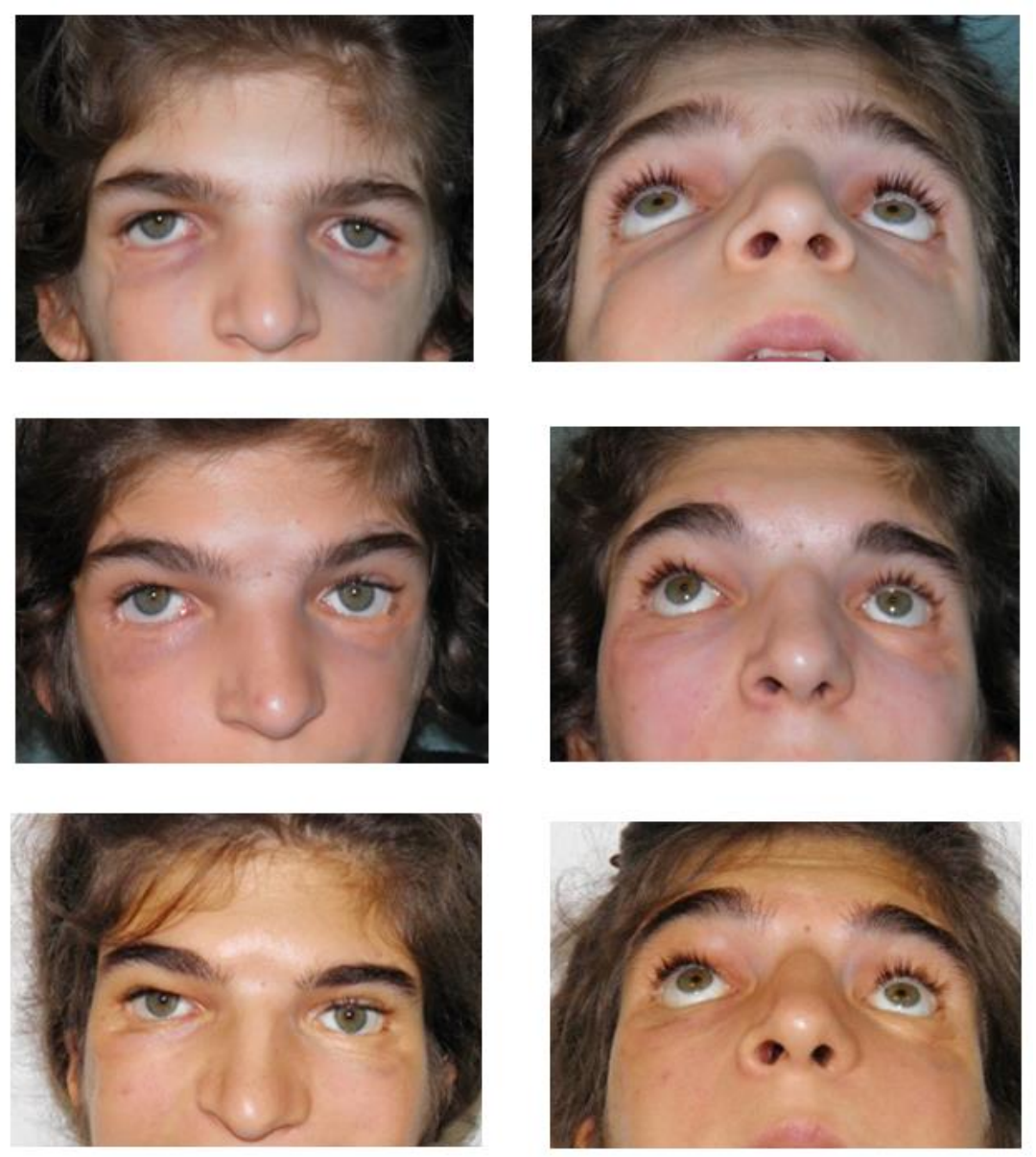

FIGURE 1,A,B,C,D,E,F Treacher Collins syndrome(A,B). At age 6 the first fat grafting procedure was performed. After 6 months total orbito-zygomatic reconstruction with cranial bone grafts according to P.Tessier. At age 8 orbito-facial skeletonization and a lack of soft tissue coverage with skin shrinking became evident(C,D). At age 10 and 11 two facial fat grafting procedures increased the soft tissue volumes three-dimensionally. The patient is shown at age 14 after one total orbital bony reconstruction and three fat grafting procedures $(E, F) C D$ 


\section{MATERIALS AND METHODS}

\section{Treatment of Treacher Collins syndrome}

The treatment of the TCS patient involves different surgical procedures over many years. In this young patient at age 6, SFG was first performed to increase the shortage of soft tissue coverage. Then , 6 months later,extensive orbitozygomatic reconstruction with cranial bone grafts was carried out. At age 8 skeletonization and relative lack of soft tissue coverage with skin shrinkage became evident. At age 10 and 11 two more facial fat grafting procedures ,following Coleman's guidelines[14] increased the soft tissue volumes three-dimensionally. The patient is shown at age 6,8 and at age 11 after one total orbital bony reconstruction and three fat grafting stages( FIGURE 1)

During the fat grafting procedures, a lipoaspirate of the patient was processed by gentle centrifugation and the resulting high-density layer (HDL)of fat was injected into the orbitalperiorbital and zygomatic areas from the periosteum,to the dermis and the skin.[15].

\section{RNA isolation}

Total RNA was isolated using the RNeasy Lipid Tissue Kit (Qiagen, Hilden, Germany), including DNase digestion with the RNase-Free DNase Set (Qiagen), from the HDL of the centrifuged lipoaspirate of the TCS patient and a healthy subject. The RNA quality and concentrations of samples were determined using the NanoDropTM ND-1000 (Thermo Scientific, MA, USA).

\section{RT2 Profiler PCR array}

For first-strand CDNA synthesis, $500 \mathrm{ng}$ of total RNA of each sample was reverse-transcribed with the RT2 First Strand Kit (Qiagen). RealTime PCR was performed according to the user manual of the Human Mesenchymal Stem Cells RT2 Profiler PCR array (SABiosciences, MD, USA) using RT2 SYBR Green ROX FAST
Mastermix (SABiosciences). This array profiles the expression of 84 key genes involved in maintaining pluripotency and self-renewal status. Thermal cycling and fluorescence detection were performed using a Rotor-Gene $Q$ 100 (Qiagen). Data were analyzed using Excelbased PCR Array Data Analysis Templates (SABiosciences). Results are reported in terms of the expression ratio of each target gene between the TCS sample and the control sample.

\section{DNA isolation}

Total DNA was isolated from blood of the TCS patient using the DNeasy Blood \& Tissue Kit (Qiagen) following the manufacturer's protocol, using overnight incubation with proteinase $\mathrm{K}$ (Qiagen). The DNA quality and concentrations of samples were determined using the NanoDropTM ND-1000.

\section{Array-CGH}

Array-CGH was performed using the Human Genome $\quad \mathrm{CGH}$ Microarray (Agilent Technologies, CA, USA) with a median probe spatial resolution of $44 \mathrm{~Kb}$ following the manufacturer's protocol. Briefly, $1 \mu \mathrm{g}$ of DNA from blood of the TCS patient (TCS sample) and $1 \mu \mathrm{g}$ of pooled sex-matched reference DNA (control sample) (Promega, WI, USA) were digested with Alul and Rsal for $2 \mathrm{~h}$ at $37^{\circ} \mathrm{C}$. After inactivation of the enzymes at $65^{\circ} \mathrm{C}$ for $20 \mathrm{~min}$, each digested sample was labeled by random priming for $2 \mathrm{~h}$ using Cy5-dUTP for sample DNA and Cy3-dUTP for control DNA. Labeled products were then column-purified (Microcon YM-30 filters, Millipore Corporation, MA, USA). After probe denaturation and preannealing with Cot-1 DNA, hybridization was performed at 65 ${ }^{\circ} \mathrm{C}$ with rotation for $24 \mathrm{~h}$. At the end of the incubation, slides were washed and analyzed using the Agilent scanner. Data and graphics were elaborated using CGH Analytics software (v3.1 Agilent Technologies).

\section{Statistical analysis}

One-way analysis of variance (ANOVA) was used for data analyses. A repeated-measures 
ANOVA with a post-hoc analysis using was calculated as the standard deviation of the Bonferroni's correction for multiple comparisons difference between measurements. All testing was performed. T-tests were used to determine was performed using SPSS 16.0 software significant differences $(p<0.05)$. Repeatability (SPSS Inc., IL, USA).
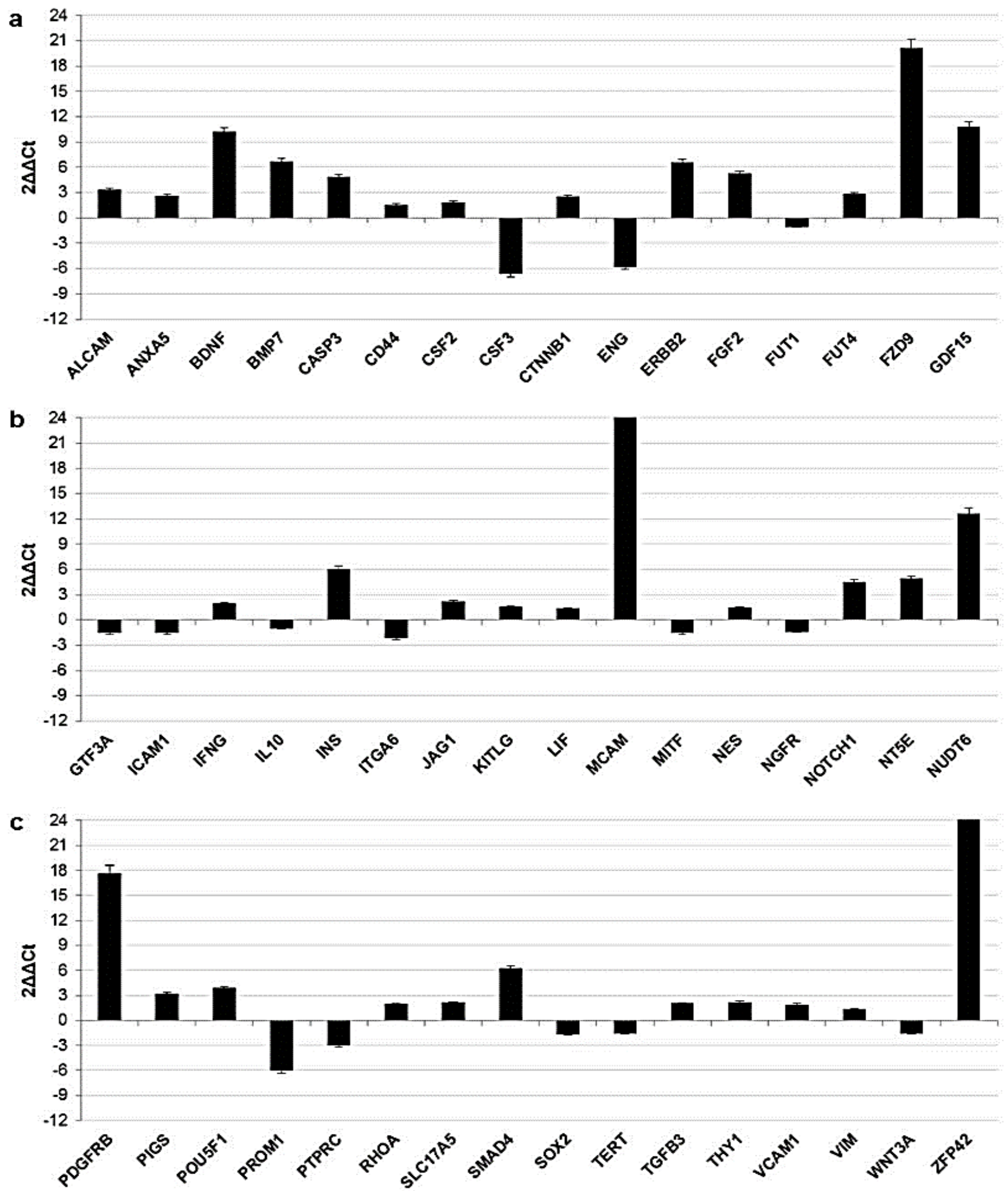

FIGURE 2(a,b,c) Gene expression profiling of MSCs-specific markers in ADSCs isolated from the HDL of fat of the TCS patient compared to those of a healthy subject. 

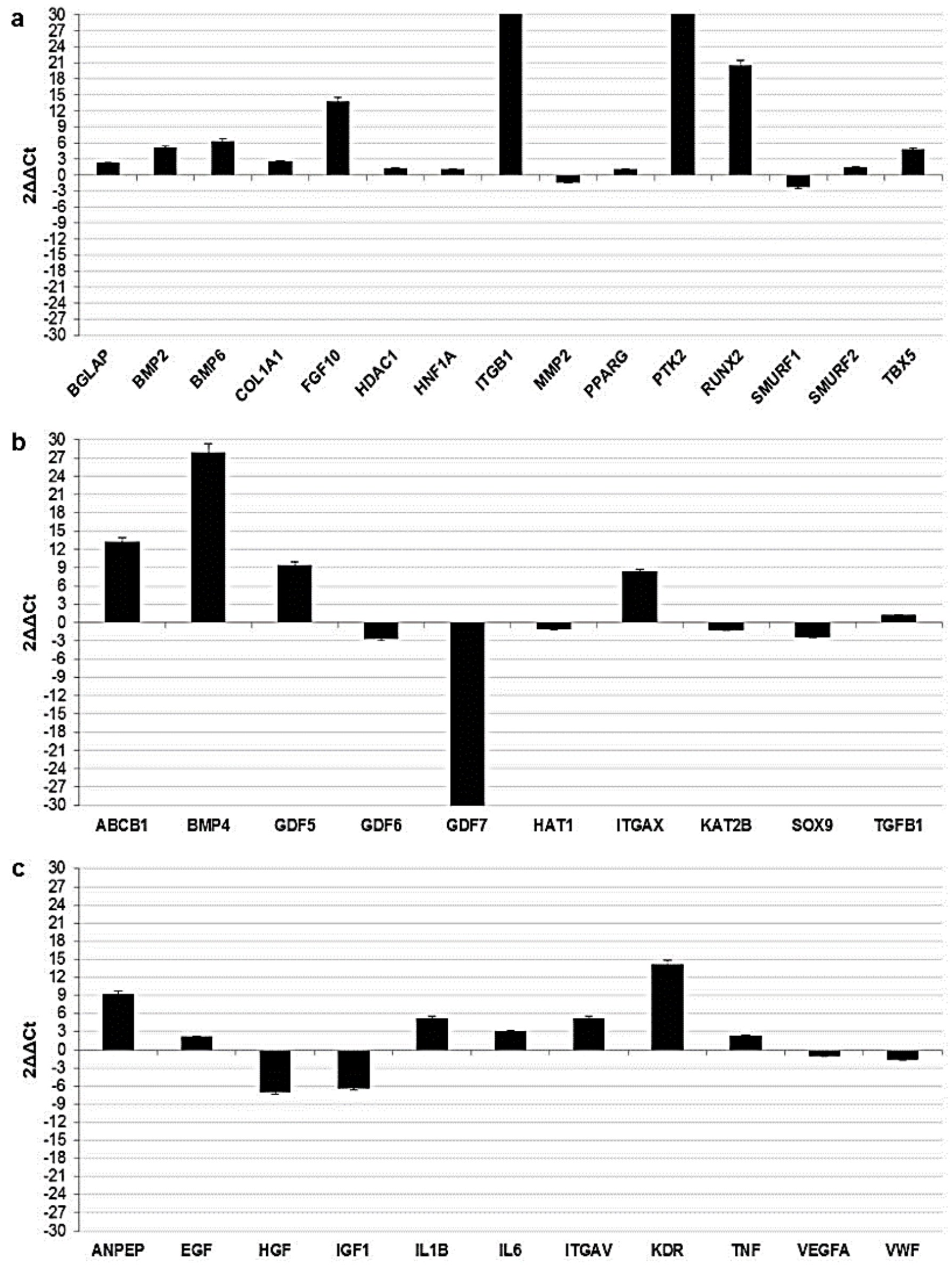

FIGURE 3 Gene expression profiling of osteogenic (a),(b)chondrogenic, and vasculogenic differentiation markers in ADSCs isolated from the HDL of fat of the TCS patient compared to those of a healthy subject(c) 
a

\begin{tabular}{|c|c|c|c|c|}
\hline Chromosome & $\begin{array}{l}\text { Chromosome } \\
\text { abnormality }\end{array}$ & $\begin{array}{l}\text { Estimated } \\
\text { size }(\mathrm{Kb})\end{array}$ & Gene & Gene full name \\
\hline 1 & Deletion q24.2 & 24 & SAC & soluble adenylyl cyclase \\
\hline 1 & Deletion q32.1 & 25 & TMCC2 & transmembrane and coiled-coil domain family 2 \\
\hline 4 & Duplication p16.1 & 49 & SORCS2 & sortilin-related VPS10 domain containing receptor 2 \\
\hline 8 & Deletion p22 & 63 & MSR1 & macrophage scavenger receptor 1 \\
\hline 11 & Deletion q13 & 17 & $\begin{array}{l}\text { MUS81 } \\
\text { EFEMP2 } \\
\text { CTSW }\end{array}$ & $\begin{array}{l}\text { MUS81 structure-specific endonuclease subunit } \\
\text { EGF containing fibulin-like extracellular matrix } \\
\text { protein } 2 \\
\text { cathepsin W }\end{array}$ \\
\hline 12 & Deletion q13.12 & 24 & SPATS2 & spermatogenesis associated, serine-rich 2 \\
\hline 12 & Deletion q13.3 & 40 & $\begin{array}{l}\text { TIMELESS } \\
\text { MIP }\end{array}$ & $\begin{array}{l}\text { timeless circadian clock } \\
\text { major intrinsic protein of lens fiber }\end{array}$ \\
\hline 19 & Duplication p13.2 & 36 & $\begin{array}{l}\text { MORG1 } \\
\text { FBXW9 } \\
\text { TNPO2 }\end{array}$ & $\begin{array}{l}\text { mitogen-activated protein kinase organizer } 1 \\
\text { F-box and WD repeat domain containing } 9 \\
\text { transportin } 2\end{array}$ \\
\hline $\mathrm{X}$ & Duplication q28 & 72 & HSFX1 & heat shock transcription factor family, $\mathrm{X}$ linked 1 \\
\hline
\end{tabular}

b

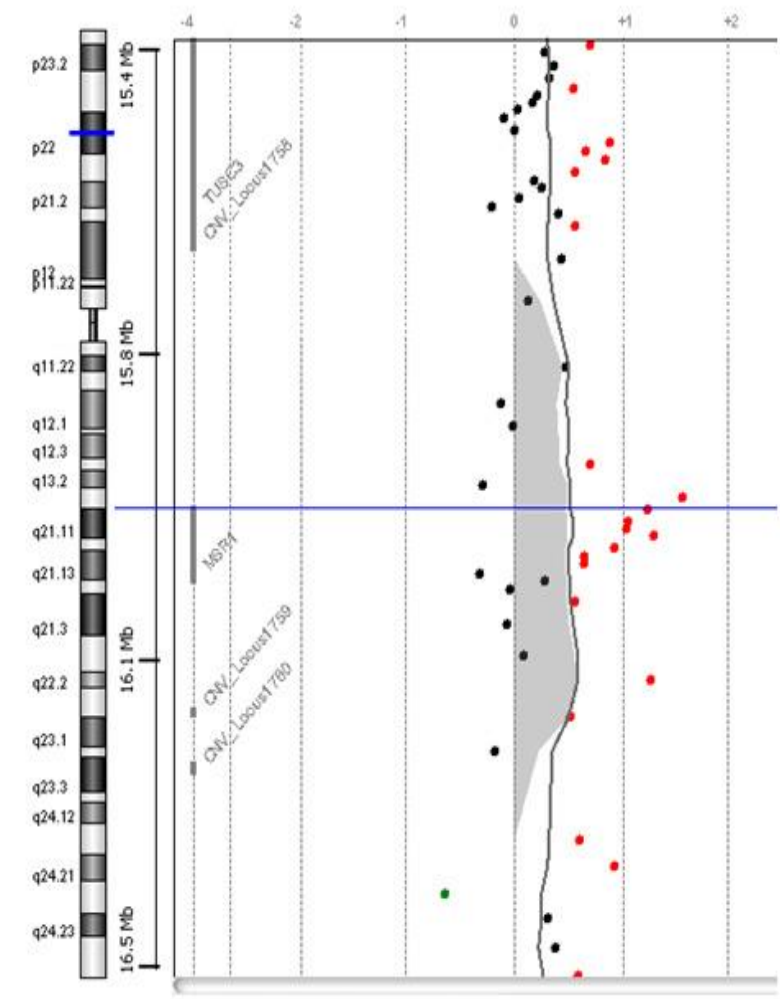

C 12: $54844261-55333150,488 \mathrm{~Kb}$

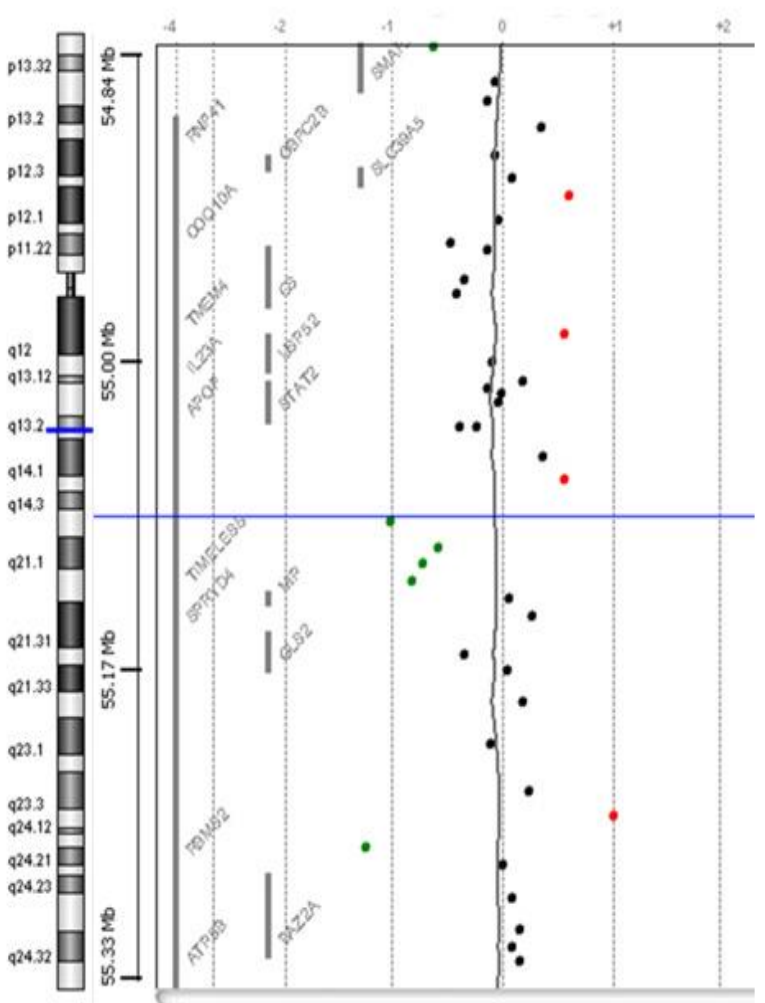

FIGURE 4 Array-CGH analysis of genomic DNA of the TCS patient. List of chromosomal alterations identified in the TCS patient with array-CGH (a). For each chromosome, the table shows the type of chromosome abnormality (deletion or amplification), estimated size of the genomic alteration, and genes localized in the involved region and their full names. Representative array-CGH profile of chromosome 8 (b) and chromosome 12, showing an amplified (red dots) and deleted (green dots) region, respectively(c). 


\section{RESULTS}

\section{Stemness properties of ADSCs}

To evaluate the stemness properties of ADSCs in the fat of the TCS patient, mRNA from the HDL was analyzed by real-time PCR. Gene expression of several mesenchymal stem cell (MSCs)-specific markers in ADSCs of the HDL of the TCS patient in comparison to those of a healthy subject(FIGURE 2). Expression of most of these genes did not significantly differ between the two populations of cells. Some genes were instead upregulated in ADSCs of the TCS patient, including genes encoding growth factors (BDNF, FGF2, GDF15, and PDGFRB), enzymes (CASP3, ERBB2, and NT5E), receptors (FZD9 and NOTCH1), signaling molecules (BMP7 and SMAD4), and other proteins (INS, MCAM, and ZFP42). Genes encoding the cytokine CSF3 and the glycoproteins ENG and PROM1 were downregulated.

Expression profiling of genes involved in osteogenic (panel a), chondrogenic (panel b), and vasculogenic (panel c) differentiation in ADSCs isolated from the TCS patient's HDL of fat. Several genes were differentially expressed between cells of the TCS patient and those of a healthy subject(FIGURE 3). These genes belong to the bone morphogenetic protein (BMP) family and transforming growth factor $\beta$ (TGF $\beta$ ) superfamily (BMP2, BMP6, BMP4, GDF5, and GDF7), growth factors (FGF10, HGF, and IGF1), members of the integrin family (ITGB1, ITGAX, and ITGAV), transcription factors (i.e., RUNX2 and TBX5), kinases (PTK2 and $\mathrm{KDR}$ ), and other proteins (ABCAB1, ANPEP, and IL1B).

\section{Analysis of genomic imbalances with array- CGH}

Array-CGH was used to rapidly screen the whole genome of the TCS patient in order to evaluate the presence of imbalanced regions. Lists the genomic regions that were altered in the TCS patient (FIGURE 4).

\section{DISCUSSION}

TCS is a rare congenital disorder of craniofacial development with bilateral midfacial zygomaticorbital bony involvement. This skeletal malformation is associated with hypoplasia of all the surrounding soft tissues layers. Although skeletal reconstruction in theory corrects the projection of the orbits and zygomas, TCS patients continue to have a soft tissue deficit. The superficial musculoaponeurotic system and periosteum are absent or extremely minimally represented. Therefore, bone grafts, even if taken from the calvaria, undergo more resorption than in normal patients. Fat grafting could increase the soft tissue facial volume, thereby supplying a valuable recipient bed for further osteoplasty [17]. Recently, it was proposed that re-establishment of soft tissues is key in the early treatment phases of TCS ${ }^{[18]}$. In line with this hypothesis, autologous fat grafting has been used as a first-line approach in the management of Parry-Romberg disease, a type of progressive hemifacial atrophy, producing satisfying and long-lasting results [19]. SFG was used as the first surgical procedure to treat the young TCS patient described here. It is speculated that SFG should be performed prior to reconstruction of bony structures because ADSCs present within the graft will stimulate subsequent bone repair by promoting osteoblast differentiation. Moreover, the adipose tissuederived stromal vascular fraction of the graft may favor early revascularization of bone grafts, promoting integration while minimizing bony resorption ${ }^{[20,21]}$. As confirmed by our recent work [15], ADSCs present in the HDL of injected fat highly express MSCs and endothelial markers, indicating a strong potential to use the cells in this layer. In the present study, we compared the stemness properties of ADSCs between the TCS patient and a healthy subject. Interestingly, there was a strong correlation between the gene expression profiles of these two populations, indicating that autologous SFG is a good strategy to treat TCS patients. Nevertheless, some genes were differentially expressed between the patient and the control sample (Fig. 
2 and 3). Most of these belonged to the BMP family and TGF $\beta$ superfamily, such as BMP2, BMP4, BMP6, BMP7, GDF5, GDF7, and GDF15. Members of these families are mediators of a wide range of biological activities in the cranial neural crest, such as cell proliferation, differentiation, and extracellular matrix formation, suggesting that BMP and TGF $\beta$ proteins are fundamental for craniofacial development [22]. Recently, gain-of-function studies indicated that elevated BMP4 activity in central neural crest results in dramatic changes in the facial skeleton ${ }^{[23]}$. TGF $\beta / B M P$ signaling reportedly relies on SMAD4-dependent pathways to mediate epithelial-mesenchymal interactions that control craniofacial organogenesis [24]. Interestingly, SMAD4 was upregulated in the HDL of fat of TCS patients. On the contrary, a significant downregulation was found for GDF7. In mouse, this gene functions as an inductive signal from the roof plate required for the specification of neuronal identity in the dorsal spinal cord. These data confirm that alterations occurring on neural crest cells are preserved during adult life in mesenchymal stem lineage. Other molecules that regulate multiple developmental processes, including craniofacial development, are fibroblast growth factors (FGF1-FGF10) [25]. Several human diseases arise from dysregulated FGF signaling, such as craniosynostosis and skeletal dysplasia syndrome [26]. In our experiments, FGF2 and FGF10 were highly expressed in ADSCs isolated from the TCS patient. BMP and FGF signaling is also involved in the expression of RUNX2, the master transcription factor for osteogenesis [27]. Later, Choi et al. discovered that FGF2 is an earlier signal than BMP2 in the cranial intramembranous bone formation process, and that RUNX2 may mediate FGF2induced BMP2 expression [28]. The NUDT6 gene is positioned on the opposite strand to the FGF2 gene. NUDT6 is an antisense gene of FGF2 in humans and rats, and may regulate the stability of FGF2 through its 3' untranslated region of
mRNA [29]. NUDT6 mRNA was upregulated in TCS patients compared with the control sample. Expression of NOTCH1 and FZD9 also significantly differed between the TCS patient and healthy subject. NOTCH1 is a receptor of the Notch family, whose members play a role in a variety of developmental processes by controlling cell fate decisions. Gain of Notch signaling in neural crest cells reportedly produces severe craniofacial malformations including cleft face and palate, exencephaly, and micrognathia as a result of deficient cranial migration and a lack of differentiation of these cells ${ }^{[30]}$. FZD9 belongs to the Frizzled family of Wnt receptors [31]. FZD9 is expressed predominantly in brain and eye, and it is involved in tissue polarity and development. ZFP42, another gene found upregulated in ADSCs of the TCS patient, is critically important in maintaining proliferative state in MSCs, while simultaneously preventing differentiation [32]. Other genes upregulated in ADSCs isolated from the TCS patient were those related to cell-cell and cellmatrix interactions, such as integrins and PTK2, a member of the focal adhesion kinase family. Interaction of cells with extracellular matrix is an essential event for differentiation, proliferation and activity of osteoblasts. In bone, binding of osteoblasts to bone matrix is required to determine specific activities of the cells and to synthesize bone matrix proteins ${ }^{[33]}$.

Taken together, our results seem to indicate that several signaling pathways, including TGF $\beta / B M P, F G F$, Notch, and Wnt signaling, are involved in the development of craniofacial structures. It is reasonable to assume that dysregulation of these complex pathways, as we observed in the gene expression experiments, is implicated in the onset of craniofacial diseases.

As stated above, due to the high variability of the resulting phenotype and the degree to which de novo mutations arise, every case of TCS is unique and needs to be assessed individually. Array-CGH was performed on the blood of the TCS patient to evaluate the presence of imbalanced regions. The main advantage of this 
technique is its ability to rapidly screen all chromosomes in a single test and to detect any DNA imbalances, including loss or gain of chromosome material, more precisely than conventional genetic analysis methods. The array-CGH results demonstrated that the DNA of the TCS patient contained several amplified or deleted regions in which genes involved in different cellular processes, such as apoptosis, embryonic development, cell cycle, DNA damage control, cell proliferation, and differentiation, are localized. In particular, we found a deletion of $40 \mathrm{~Kb}$ in $12 \mathrm{q} 13.3$, which contains the TIMELESS gene. In mammals, TIMELESS controls the circadian rhythm and its expression exhibits a 24 hour oscillation [34]. Genetic studies showed that TIMELESS is essential for early embryonic development; its homozygous knockout leads to early embryonic lethality in mice ${ }^{[35]}$. A recent study demonstrated that TIMELESS also plays a key role in the regulation of embryonic stem cell differentiation [36]. One of the earliest steps in embryonic development is the formation of the proamniotic cavity, which involves the coordinated apoptosis of embryonic cells. Embryonic cells lacking TIMELESS have reduced caspase activity, and fail to undergo programmed cell death during cavitation and remain pluripotent. These findings raise the possibility that circadian rhythms and early development are intimately linked. TIMELESS also appears to be linked to the cell cycle and DNA damage control [37], as well as the gene MUS81. In the DNA of the TCS patient, we found a deletion of $17 \mathrm{~Kb}$ in $11 \mathrm{q} 13$, where the MUS81 gene is localized. The endonuclease MUS81 plays an important role in determining the rate of DNA replication and S-phase progression in human cells ${ }^{[38]}$. MUS81 is important for DNA damage repair and genome integrity in murine and human cells [39]. In addition, we found an amplification in 4p16.1, where the gene SORCS2 is localized. A study of murine embryos demonstrated that the receptor SORCS2 is involved in embryonic development [40]. Expression of this protein is high and transient in derivatives of all three germ layers. In particular, high amounts of SORCS2 protein are expressed in the facial mesenchyme, a derivative of the neural crest, up to 16.5 days of murine embryonic development.

The data collected may represent a starting point for the identification of new strategies to treat TCS. We found that some alterations, usually associated to embryonic stem cells, are preserved in MSCs and conserved during adult life. Consequently, with the aim to improve the final results, we need to consider as a fundamental step combining knowledge of genetic alterations and expression profiles before starting with surgical procedures.

\section{Acknowledgements}

This research was supported by a University of Padova grant to Barbara Zavan (Progetto di Ateneo)

\section{DECLARATIONS}

Authors'Contributions: Barbara Zavan, Luigi Clauser conceived and designed the experiments; Luigi Clauser, Antonio Lucchi performed surgical treatments and samples collection; Chiara Gardin, Letizia Ferroni performed the experiments; Chiara Gardin,Barbara Zavan analyzed the data; Barbara Zavan, Luigi Clauser contributed reagents/materials/analysis tools; Chiara Gardin, Letizia Ferroni, Barbara Zavan, Luigi Clauser, Carolina Sannino, Maria Elena de Notariis prepared the article.

\section{Availability of data and materials}

Not applicable

\section{Financial support and sponsorship}

None

\section{Conflicts of interest}

All Authors declared that there are no conflicts of interest

\section{Ethical approval and consent to participate}


Not applicable

\section{Consent for publication}

Written informed consent was obtained for all patient images.

\section{Copyright}

CThe Authors (2021)

\section{REFERENCES}

[1]. Treacher Collins E. Case with symmetrical congenital notches in the outer part of each lower lid and defective development of the malar bones. Trans Ophthalmol Soc UK 1900; 20:190193

[2]. Sakai D, Trainor PA. Treacher Collins syndrome: unmasking the role of Tcof $1 /$ treacle. Int $\mathrm{J}$ Biochem Cell Biol 2009;41:1229-1232

[3]. Poswillo D. The pathogenesis of the Treacher Collins syndrome (mandibulofacial dysostosis). Br J Oral Surg 1975;13:1-26

[4]. Passos-Bueno M, Ornelas CC, Fanganiello RD. Syndromes of the first and second pharyngeal arches: A review. Am $J$ Med Genet $A$ 2009;149A:1853-1859

[5]. Dixon J, Jones NC, Sandell LL, et al. Tcof $1 /$ Treacle is required for neural crest cell formation and proliferation deficiencies that cause craniofacial abnormalities. Proc Natl Acad Sci U S A 2006;103:13403-13408

[6]. Dixon MJ, Marres HA, Edwards SJ, et al. Treacher Collins syndrome: correlation between clinical and genetic linkage studies. Clin Dysmorphol 1994;3:96-103

[7]. Treacher Collins Syndrome Collaborative Group. Positional cloning of a gene involved in the pathogenesis of Treacher Collins syndrome. Nat Genet 1996;12:130-136

[8]. Valdez BC, Henning D, So RB, et al. The Treacher Collins syndrome (TCOF1) gene product is involved in ribosomal DNA gene transcription by interacting with upstream binding factor. Proc Natl Acad Sci $U$ S A 2004;101:10709-10714

[9]. Jones NC, Lynn ML, Gaudenz K, et al. Prevention of the neurocristopathy Treacher Collins syndrome through inhibition of p53 function. Nat Med 2008;14:125-133

[10]. Splendore A, Jabs EW, Félix TM, et al. Parental origin of mutations in sporadic cases of Treacher Collins syndrome. Eur $J$ Hum Genet 2003;11:718-722
[11]. Trainor PA, Dixon J, Dixon MJ. Treacher Collins syndrome: etiology, pathogenesis and prevention. Eur J Hum Genet 2009;17:275-283

[12]. Arndt EM, Travis F, Lefebvre A, et al. Psychosocial adjustment of 20 patients with Treacher Collins syndrome before and after reconstructive surgery. $\mathrm{Br} J$ Plast Surg 1987;40:605-609

[13]. Clauser L, Tieghi R, Mandrioli S, et al. Facial lipostructure in complex reconstructive surgery. Riv Ital Chir Plast 2005;37:75-79

[14]. Coleman SR. Structural fat grafting: more than a permanent filler. Plast Reconstr Surg 2006;118:108S-120S

[15]. Clauser L, Ferroni L, Gardin C, et al. Selective augmentation of stem cell populations in structural fat grafts for maxillofacial surgery. PLoS One 2014;9:e110796

[16]. Coleman SR. Structural fat grafting. Aesthet Surg J 1998;18:386-388

[17]. Clauser LC. Optimizing maxillofacial and craniofacial results. In: Coleman SR, Mazzola RF. eds. Fat Injection: From Filling to Regeneration. Thieme Medical, 2009:475-500

[18]. Herlin C, Doucet JC, Bigorre M, et al. Computerassisted midface reconstruction in Treacher Collins syndrome part 2: soft tissue reconstruction. J Craniomaxillofac Surg 2013;41:676-680

[19]. Clauser LC, Tieghi R, Consorti G. ParryRomberg syndrome: volumetric regeneration by structural fat grafting technique. $J$ Craniomaxillofac Surg 2010;38:605-609

[20]. Lee K, Kim H, Kim JM, et al. Systemic transplantation of human adipose-derived stem cells stimulates bone repair by promoting osteoblast and osteoclast function. $J$ Cell Mol Med 2011;15:2082-2094

[21]. Scherberich A, Muller AM, Schafer DJ, et al. Adipose tissue-derived progenitors for engineering osteogenic and vasculogenic grafts. J Cell Physiol 2010;225:348-353

[22]. Sasaki T, Ito $Y$, Bringas $P$ Jr, et al. TGFbetamediated FGF signaling is crucial for regulating cranial neural crest cell proliferation during frontal bone development. Development 2006;133:371381

[23]. Bonilla-Claudio M, Wang J, Bai $\mathrm{Y}$, et al. Bmp signaling regulates a dose-dependent transcriptional program to control facial skeletal development. Development 2012;139:709-719 
[24]. Xu X, Han J, Ito Y, et al. Ectodermal Smad4 and p38 MAPK are functionally redundant in mediating TGF-beta/BMP signaling during tooth and palate development. Dev Cell 2008;15:322329

[25]. Ornitz DM; Itoh N. Fibroblast growth factors. Genome Biol 2001,2:REVIEWS3005

[26]. Riley BM, Mansilla MA, Ma J, et al. Impaired FGF signaling contributes to cleft lip and palate. Proc Natl Acad Sci U S A 2007;104:4512-4517

[27]. Komori T, Yagi $H$, Nomura $S$, et al. Targeted disruption of Cbfa1 results in a complete lack of bone formation owing to maturational arrest of osteoblasts. Cell 1997;89:755-764

[28]. Choi KY, Kim HJ, Lee MH, et al. Runx2 regulates FGF2-induced Bmp2 expression during cranial bone development. Dev Dyn 2005;233:115-121

[29]. Li AW, Murphy PR. Expression of alternatively spliced FGF-2 antisense RNA transcripts in the central nervous system: regulation of FGF-2 mRNA translation. Mol Cell Endocrinol 2000;162:69-78

[30]. Mead TJ, Yutzey KE. Notch pathway regulation of neural crest cell development in vivo. Dev Dyn 2012;241:376-389

[31]. Wang YK, Spörle R, Paperna $T$, et al. Characterization and expression pattern of the frizzled gene Fzd9, the mouse homolog of FZD9 which is deleted in Williams-Beuren syndrome. Genomics 1999;57:235-248

[32]. Bhandari DR, Seo KW, Roh KH, et al. REX-1 expression and p38 MAPK activation status can determine proliferation/differentiation fates in human mesenchymal stem cells. PLoS One 2010;5:e10493

[33]. Demais V, Audrain C, Mabilleau G, et al. Diversity of bone matrix adhesion proteins modulates osteoblast attachment and organization of actin cytoskeleton. Morphologie 2014;98:53-64

[34]. Barnes JW, Tischkau SA, Barnes JA, et al. Requirement of mammalian Timeless for circadian rhythmicity. Science 2003;302:439-442

[35]. Gotter AL, Manganaro T, Weaver DR, et al. A time-less function for mouse timeless. Nat Neurosci 2000;3:755-756

[36]. O'Reilly LP, Watkins SC, Smithgall TE. An unexpected role for the clock protein timeless in developmental apoptosis. PLOS One 2011;6:e17157

[37]. Unsal-Kaçmaz K, Mullen TE, Kaufmann WK, et al. Coupling of human circadian and cell cycles by the timeless protein. $\mathrm{Mol}$ Cell Biol 2005;25:3109-3116

[38]. Fu H, Martin MM, Regairaz M, et al. The DNA repair endonuclease Mus81 facilitates fast DNA replication in the absence of exogenous damage. Nat Commun 2015;6:6746

[39]. El Ghamrasni S, Cardoso R, Halaby MJ, et al. Cooperation of Blm and Mus81 in development, fertility, genomic integrity and cancer suppression. Oncogene 2015;34:1780-1789

[40]. Rezgaoui M, Hermey G, Riedel IB, et al. Identification of SorCS2, a novel member of the VPS10 domain containing receptor family, prominently expressed in the developing mouse brain. Mech Dev 2001;100:335-338

By using the site/services, you are agreeing to our Policies: https://escipub.com/terms-privacy-policy-disclaimer/ 